Sarah I. Tarbox

BS, Brandeis University, 1997

Submitted to the Graduate Faculty of

Arts and Science in partial fulfillment

of the requirements for the degree of

Master of Science

University of Pittsburgh 


\section{UNIVERSITY OF PITTSBURGH}

\section{FACULTY OF ARTS AND SCIENCES}

This thesis was presented

by

Sarah I. Tarbox

It was defended on

January 24, 2005

and approved by

Michael F. Pogue-Geile, Ph.D.

Thesis Advisor

Jeffrey F. Cohn, Ph.D.

Julie Fiez, Ph.D.

Karen L. Schmidt, Ph.D. 


\title{
SPONTANEOUS DYSKINESIA AND FAMILIAL LIABILITY TO SCHIZOPHRENIA
}

\author{
Sarah I. Tarbox, MS \\ University of Pittsburgh, 2005
}

\begin{abstract}
Several factors suggest that spontaneous dyskinesia may be a useful non-diagnosis phenotype for further elucidating the specific nature of the genetic contribution to schizophrenia. For example, involuntary movement abnormalities have been observed in both medicated and unmedicated schizophrenia patients, in individuals with schizotypal personality disorder, and sometimes in siblings of schizophrenia patients. However, there are many inconsistencies present in the literature to date. The current study thus sought to investigate the existence of spontaneous dyskinesia in schizophrenia patient probands, their non-psychotic siblings, and healthy controls in order to clarify its potential value as an "endophenotypes" in genetic studies of schizophrenia. Videotaped interviews were coded for the presence of spontaneous, involuntary movement abnormalities by a trained and reliable rater who was blind to group and family status. The results of this study indicated that siblings of schizophrenia patients did not display significantly more involuntary movements compared to controls, although tremor was observed in a few siblings. In contrast, schizophrenia patients did display significantly more involuntary movements compared to controls as well as their non-psychotic siblings. The lack of significant differences between siblings and controls argues against a strong association between spontaneous dyskinesia and an underlying genetic liability to schizophrenia. Thus, it appears that involuntary movement abnormalities may be limited in their utility as endophenotypes in genetic studies of schizophrenia, despite being associated with schizophrenia itself.
\end{abstract}




\section{TABLE OF CONTENTS}

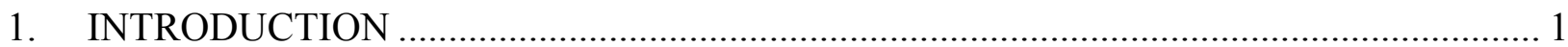

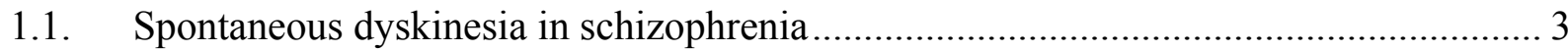

1.2. Spontaneous dyskinesia in schizophrenia spectrum disorders...................................... 4

1.3. Spontaneous dyskinesia in relatives of schizophrenia patients...................................... 5

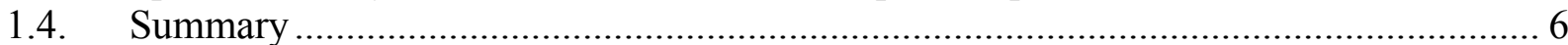

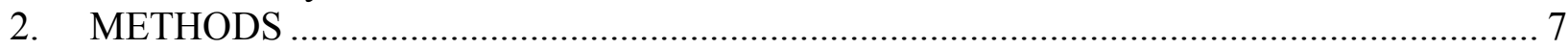

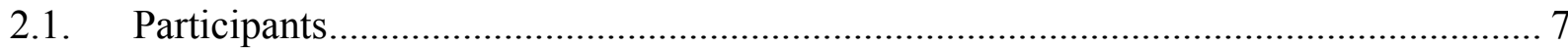

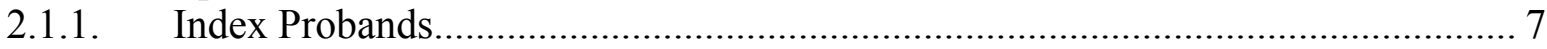

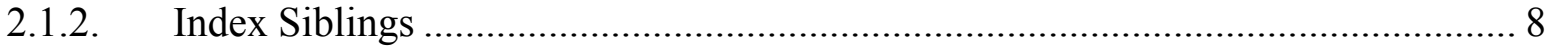

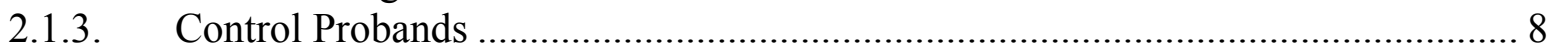

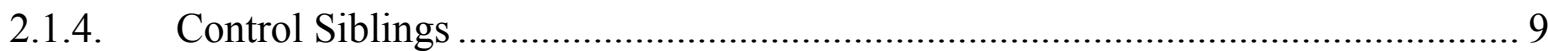

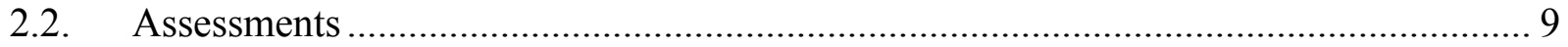

2.2.1. General Psychopathology ....................................................................... 9

2.2.2. Spontaneous Dyskinesia ………………………………………………....... 10

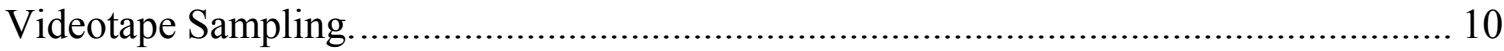

Spontaneous Dyskinesia Coding..................................................................................... 10

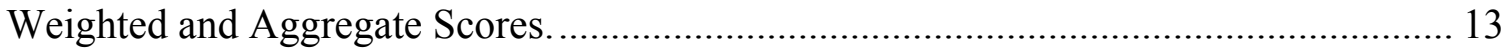

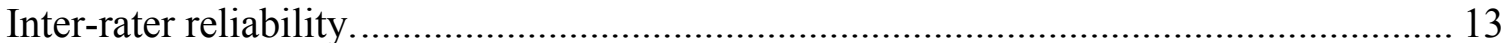

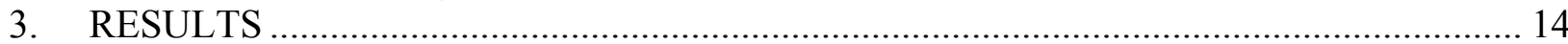

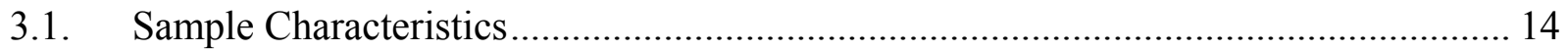

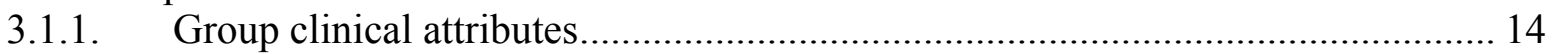

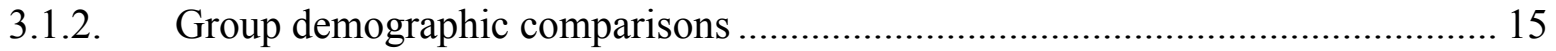

3.2. Spontaneous Dyskinesia …………………....................................................... 17

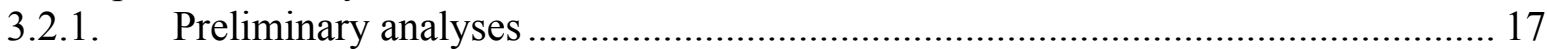

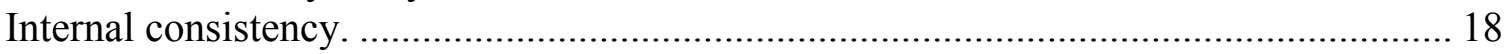

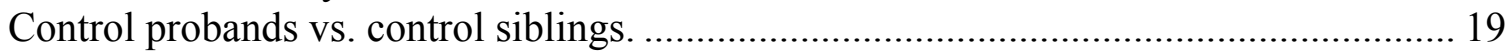

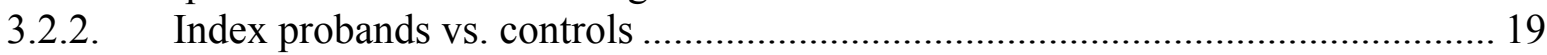

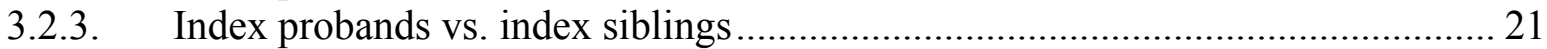

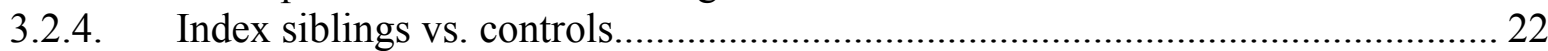

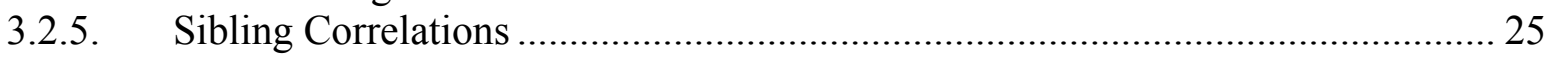

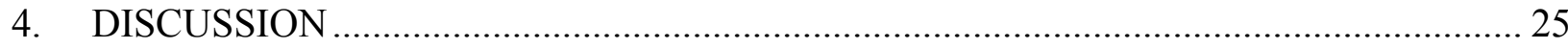

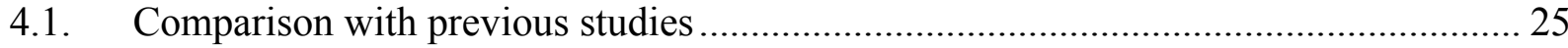

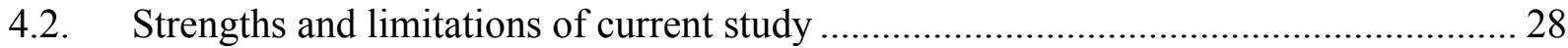

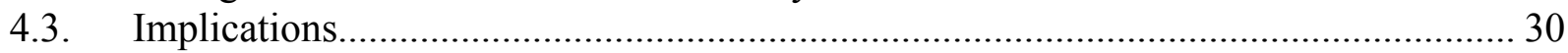

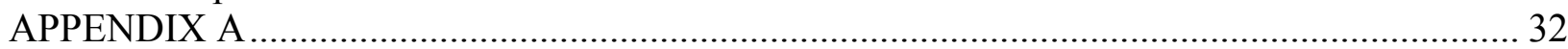

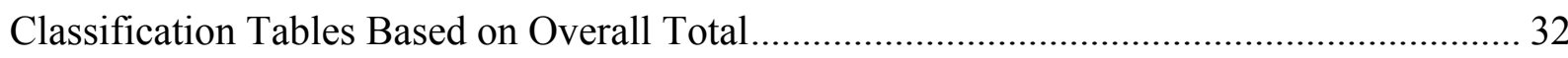

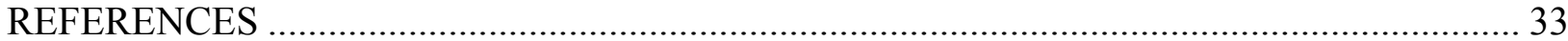




\section{LIST OF TABLES}

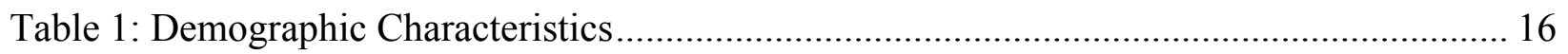

Table 2: Spontaneous Dyskinesia ................................................................................ 18

Table 3: Pairwise Group Analyses: Overall Total and Movement Categories.......................... 24 


\section{INTRODUCTION}

After decades of research, the specific nature of the genetic contribution to schizophrenia remains unclear. Although family, twin, and adoption studies indicate that the diagnosis of schizophrenia is itself highly heritable [estimated at approximately .80 (Cardno et al., 1999)], and despite some promising leads, the search for specific genetic abnormalities has not yet clearly identified any genes of major or moderate effect (O'Donovan et al., 2003). This suggests that several genes affect the diagnosis of schizophrenia, but the diagnosis is itself not highly associated with any individual gene variant (Pogue-Geile \& Gottesman, 1999).

One possible means of making progress in this area is to identify non-diagnosis phenotypes that are more associated with specific genetic liabilities than is the schizophrenia diagnosis itself. In seeking to identify such “endophenotypes”(Gottesman \& Gould, 2003; Gottesman \& Shields, 1972) an initial rule of thumb is that a candidate phenotype should be more frequently observed in individuals with schizophrenia than controls and should not be an artifact that is secondary to having the disorder (e.g., an effect of taking antipsychotic medication). The greater the association between a candidate phenotype and the putative pathology of schizophrenia, the more useful it may be. Once this association is established, the presence and nature of the phenotype in relatives of schizophrenia patients should be evaluated. The more strongly the phenotype is associated with the genetic liability to the disorder, evidenced by either increased frequency or severity in relatives compared to non-relatives, the greater its potential value.

Spontaneous dyskinesia appears to be a plausible candidate for use with this strategy. Spontaneous dyskinesia refers to abnormal, involuntary movements of the face, limbs, or trunk that occur unprompted and are not under the control of the individual. In this report, the terms 
"spontaneous dyskinesia" and "spontaneous involuntary movement abnormality" will be used interchangeably.

Several factors suggest that spontaneous dyskinesia may be a useful endophenotype for further elucidating the nature of genetic effects in schizophrenia. Foremost, there are numerous reports in the literature of involuntary movement abnormalities in both unmedicated and medicated schizophrenia patients. Secondly, involuntary movements have been observed in individuals with schizophrenia spectrum disorders (e.g., schizotypal, schizoid, or paranoid personality disorder). This group of individuals has been hypothesized to share genetic liability with schizophrenia. Thirdly and most importantly, there is also evidence that movement abnormalities may be present in the non-psychotic relatives of schizophrenia patients. These three areas will be reviewed briefly below.

Before considering these, it is worth noting that many involuntary movement abnormalities can be produced by damage to, or dysregulation of specific brain regions and related neurotransmitter systems that also may be implicated in schizophrenia. For example, lesions involving the caudate nucleus, putamen, globus pallidus, subthalamic nucleus, thalamus, and cerebellum have been associated with particular spontaneous dyskinesias in several studies using a variety of methodological techniques (e.g., Antonini et al., 1998; Dooling \& Adams, 1975; Kim et al., 2002; Krystkowiak et al., 2000; Krystkowiak et al., 1998; LeDoux \& Brady, 2003; Lehericy et al., 1996; Lenz et al., 2002; Lozza et al., 2002; Naumann et al., 1996; Perlmutter et al., 1997). Moreover, an array of structural and functional abnormalities have been observed in several of these regions in individuals with schizophrenia, although substantial variation in findings exists (e.g., Danos et al., 2002; Gunduz et al., 2002; Jernigan et al., 1991; Keshavan et al., 1998; Lang et al., 2001; Marcelis et al., 2003; McCreadie et al., 2002; Menon et 
al., 2001; Muller et al., 2002; Sigmundsson et al., 2001; Staal et al., 2000; Staal et al., 2001; Young et al., 2000). Thus, to the extent that it has been demonstrated in individuals with spontaneous dyskinesia and in individuals with schizophrenia, the presence of pathology in brain regions integral to normal movement control is consistent with a connection between movement abnormalities and schizophrenia.

\subsection{Spontaneous dyskinesia in schizophrenia}

As outlined above, the first step in evaluating the potential utility of spontaneous dyskinesia is to establish the frequency and characteristics of abnormal movements in individuals with schizophrenia and determine whether or not they are simply artifacts. The primary concern is with the effects of medication, as there is evidence that neuroleptics can produce movement abnormalities (e.g., extrapyramidal symptoms, tardive dyskinesia) (Crane, 1968; Marsden \& Jenner, 1980), as well as neurotoxicity, volumetric abnormalities, and changes in blood flow that may alter the functioning of brain regions associated with movement regulation (e.g., the basal ganglia) (Corson et al., 2002; Gil-ad et al., 2001; Gunduz et al., 2002). Given this concern, only findings pertaining to spontaneous involuntary movements reported in neuroleptic naïve schizophrenia patients (individuals who have never been exposed to neuroleptic medication) will be reviewed here.

Ten studies have investigated the presence of spontaneous dyskinesia in individuals with schizophrenia who are neuroleptic naïve (Caligiuri et al., 1993; Chatterjee et al., 1995; Chorfi \& Moussaoui, 1989; Fenn et al., 1996; Gervin et al., 1998; McCreadie \& Ohaeri, 1994; McCreadie et al., 1996; Owens et al., 1982; Puri et al., 1999; Srinivasan et al., 2001), and seven of these studies observed some spontaneous dyskinesia in these patients, including tremor, choreoathetosis, dystonia, and akathisia. The average percentage of spontaneous dyskinesia in 
schizophrenia patients reported by these studies, weighted by sample size, was $16 \%-25 \%$ depending on the stringency of the criteria employed. It is possible that age may be positively associated with the frequency of these abnormal movements, as the studies that included patient samples over 60 years of age reported the highest percentages of spontaneous dyskinesia.

Of the three studies that included a control group, one study demonstrated a greater percentage of spontaneous dyskinesia in neuroleptic naïve patients compared to controls, and the other two studies reported that neither controls nor patients demonstrated abnormal movements. The average percentage of spontaneous dyskinesia in controls, weighted by sample size, was $9 \%-12 \%$. Thus, it appears that in a substantial minority of patients, spontaneous dyskinesia may be associated with the diagnosis of schizophrenia and may not be simply an artifact of medication, although further study is required given the methodological constraints of these studies and the inconsistent results.

\subsection{Spontaneous dyskinesia in schizophrenia spectrum disorders}

To date, schizotypal personality disorder (SPD) has received the most support in the literature for its potential genetic relatedness to schizophrenia (Pogue-Geile, in press). Two studies have reported on spontaneous dyskinesia in schizotypal individuals. Cassady et al. (1998) reported higher ratings of dyskinesia in SPD participants compared to individuals with schizoid personality and normal controls, with $24 \%$ of schizotypal participants being diagnosed with dyskinesia based on a global rating score. Walker et al. (1999) also reported that SPD participants demonstrated significantly more involuntary movements, particularly of the head, arms, hands, and fingers, compared to individuals with another personality disorder and nonpersonality disordered controls. If individuals with SPD do share a portion of the genetic liability to schizophrenia, then these findings suggest a connection between movement 
abnormalities and susceptibility to the disorder. However, much remains unknown regarding the degree of genetic correlation between schizophrenia spectrum disorders and schizophrenia itself, as well as the relative risk of acquiring schizophrenia once being diagnosed with a spectrum disorder (e.g., MacDonald et al., 2001).

\subsection{Spontaneous dyskinesia in relatives of schizophrenia patients}

The extent to which these movement abnormalities are more frequently observed in relatives of schizophrenia patients compared to controls constitutes a crucial piece of evidence that spontaneous dyskinesia may be associated with the genetic liability to the disorder. We are aware of four studies to date that have reported on the percentage of spontaneous involuntary movements in non-psychotic, adult, first-degree relatives (parents and siblings) of individuals with schizophrenia compared with controls (Chen et al., 2000; Flyckt et al., 1999; Kinney et al., 1986; McCreadie et al., 1996). Three of the four studies observed some spontaneous dyskinesia in the first-degree relatives (e.g., tremor, chorea), and two of these reported a higher percentage of spontaneous dyskinesia in relatives compared to controls. The two studies that included relatives with a mean age in the 60 s reported the highest percentage of movement abnormalities, thus age may have affected the results. However, there are exceptions to this pattern (Kinney et al., 1986). The average percentage of spontaneous dyskinesia in relatives, weighted by sample size, was $19 \%-24 \%$ depending on the criteria employed, whereas the weighted average in controls was $11 \%$.

The above results suggest that spontaneous dyskinesia may be present in a subset of relatives of schizophrenia patients. However, these findings are not entirely persuasive given the inadequacies of the current literature. Not only are there few studies on spontaneous dyskinesia in relatives, there are several methodological limitations. For example, exclusion criteria for the 
relatives varied considerably across these studies: Chen et al. (2000) excluded any mental illness, Kinney et al. (1986) excluded schizophrenia, Flyckt et al. (1999) excluded any psychotic disorder, and McCreadie et al. (1996) did not report exclusion criteria. Furthermore, within each study, exclusion criteria for the control groups were often stricter than that for the relatives. For example, Flyckt et al. (1999) and Kinney et al. (1986; Woods et al., 1986) excluded controls with any psychiatric disorder. In addition, although all of the studies used standardized rating scales to assess abnormal movement, most of the scales used were not specifically designed to assess spontaneous and involuntary movement abnormalities. Moreover, only two of the four studies (Kinney et al., 1986; McCreadie et al., 1996) reported that the assessments were conducted blind to participants' group status.

\subsection{Summary}

Taken together, these findings may suggest that spontaneous, involuntary movement abnormalities exist in a substantial minority of neuroleptic naïve schizophrenia patients, schizotypal individuals, and perhaps the first-degree relatives of schizophrenia patients. Additionally, such movements also have potentially interesting brain correlates. This evidence suggests that spontaneous dyskinesia may be a useful non-diagnosis phenotype in the search for specific genetic abnormalities in schizophrenia. However, despite this apparent promise, there are considerable inconsistencies and confounds present in the literature. Further investigation appears warranted to resolve these methodological problems and clarify the value of spontaneous dyskinesia in genetic research on schizophrenia.

For these reasons, this study sought to determine the existence of spontaneous dyskinesia in siblings of schizophrenia or schizoaffective probands using sensitive, reliable, and blind 
assessments, while minimizing confounds (e.g., age, control group selection criteria) that previously existed in the literature. This study tested the following hypotheses:

1) Schizophrenia/schizoaffective patient probands display more involuntary movements compared to controls;

2) Schizophrenia/schizoaffective patient probands display more involuntary movements compared to their siblings;

3) Index siblings display more involuntary movements compared to controls.

In addition, this study investigated which categories of involuntary movements are most prominent and which upper body regions evidence involuntary movements the most.

\section{METHODS}

\subsection{Participants}

Spontaneous dyskinesia was assessed in four groups of participants: schizophrenia or schizoaffective index probands, their biological index siblings, demographically matched well control probands, and their biological control siblings. All individuals were participants in the University of Pittsburgh Sibling Study of Schizophrenia and Neuropsychology (e.g., Craver \& Pogue-Geile, 1999).

\subsubsection{Index Probands}

Schizophrenia or schizoaffective outpatients were recruited from a clinic specializing in the treatment of schizophrenia at the Western Psychiatric Institute and Clinic of the University of Pittsburgh. Screening criteria were a chart diagnosis of schizophrenia or schizoaffective disorder; English as a first language; age between 18 and 45 years; at least one full biological sibling living in the greater Pittsburgh area; no history of diagnosed neurological disease; no history of alcohol or substance abuse that would put the diagnosis of schizophrenia into question; minimum of 6 months since last hospital discharge; and current symptom stability as judged by 
clinicians. All patients who met the above criteria, gave informed consent for their own participation, and gave permission to contact at least one of their full biological siblings, were interviewed with the Schedule for Affective Disorders and Schizophrenia - Lifetime Version (SADS-L) (Endicott \& Spitzer, 1978) to confirm that they met the Research Diagnostic Criteria (RDC) (Spitzer et al., 1978) for a lifetime definite diagnosis of schizophrenia or schizoaffective disorder.

\subsubsection{Index Siblings}

One full biological sibling was recruited for each participating index patient proband. To include siblings who were matched demographically as closely as possible to the patients, siblings were ranked and recruited based on their similarity in sex and age to their patient relative, with sex similarity having the higher priority. Only siblings without RDC diagnoses of schizophrenia, schizoaffective disorder, or neurological disease were included in analyses. Otherwise, the psychopathology of the patient siblings was not constrained.

\subsubsection{Control Probands}

Healthy control probands were recruited through notices in a weekly mailed advertising publication. Initial inclusion criteria, determined through a telephone interview, were: English as a first language; age between 18 and 45 years; no personal history of psychiatric treatment; no history of diagnosed neurological disease; no history of schizophrenia or psychosis among firstdegree relatives; and a least one full biological sibling in the greater Pittsburgh area interested in participating in the study. Attempts were also made to select respondents who as a group matched the siblings of the schizophrenia probands on sex, age, ethnicity, and years of education. Individuals meeting these initial criteria were further screened over the telephone with a modified version of the SADS-L for any probable RDC diagnosis and with a modified version of 
the Structured Interview for DSM-III Personality Disorders (SIDP) (Pfohl et al., 1982) for schizoid, schizotypal, and paranoid personality disorders before in-person interviews.

\subsubsection{Control Siblings}

One full biological sibling of each healthy control proband was recruited. Similar to the patient siblings, control siblings were ranked and recruited based on their sex- and age-match to their relative. The only exclusion criteria were: any personal lifetime history of RDC schizophrenia, schizoaffective disorder, or diagnosed neurological disease. All other characteristics of these siblings were free to vary.

\subsection{Assessments}

\subsubsection{General Psychopathology}

Trained research associates or graduate students in clinical psychology interviewed all participants in person with the SADS-L, and hospital chart information was collected if available. Additionally, all participants except the schizophrenia patients were interviewed with the complete SIDP to assess the presence of DSM-III Axis II personality disorders. RDC diagnoses and DSM-III Axis II diagnoses derived from the SADS-L and SIDP, respectively, were made by a diagnostic team chaired by an experienced licensed clinical psychologist (M. P.-G.) who trained on the SIDP with its developers at the University of Iowa. The chair of the diagnostic team was blind to group membership, although the interviewers were not.

The RDC diagnoses derived from the SADS-L and SIDP were grouped into four categories: 'schizophrenia', 'schizophrenia spectrum', 'other', and 'non-diagnosis'. The schizophrenia category included individuals who endorsed schizophrenia episodes only, schizoaffective episodes only, or both schizophrenia and schizoaffective episodes. The 
schizophrenia spectrum category consisted of those individuals who received a diagnosis of schizotypal personality disorder, schizoid personality disorder, or paranoid personality disorder. The other category included individuals who received a diagnosis other than schizophrenia, schizoaffective disorder, or a schizophrenia spectrum diagnosis, including mania, depression, alcoholism, substance abuse, panic disorder, generalized anxiety disorder, obsessive compulsive disorder, specific phobia, or a personality disorder other than paranoid, schizoid, or schizotypal personality disorder. The non-diagnosis category included those individuals who did not receive any diagnosis.

\subsubsection{Spontaneous Dyskinesia}

Each participant meeting the above inclusion criteria, and for whom videotape of the inperson clinical interview was available, was included in the present study. Each of these participants was videotaped during the administration of the SADS-L and (except for the schizophrenia patients) the complete SIDP. Participants were seated during the interview. The video camera was located behind the interviewer and positioned to keep the upper body of the participant in view.

Videotape Sampling. The first 30 minutes from each videotaped interview was selected for coding. In order to analyze the video accurately and efficiently, each 30-minute segment was converted into an MPEG1 file and stored on a CD-ROM prior to viewing. The video segments were assigned arbitrary study numbers unassociated with group or family status, were viewed in a randomized order, and were coded without audio to maintain the blind.

Spontaneous Dyskinesia Coding. Each segment was assessed for the presence of spontaneous, involuntary movement abnormalities by a trained coder who was blind to group and family status. The same individual coded all participants. (A detailed description of the 
coding procedures is available from the author.) Based on previous research (e.g., Walker et al., 1999), only movement abnormalities affecting the upper limbs, head, face, and trunk of each participant were assessed. In the context of this study, "spontaneous" refers to movements that did not occur in response to a command (e.g., not elicited by an examiner asking the individual to perform a task). The term "involuntary" refers to movements that did not appear to be intentionally initiated by the participant and occurred seemingly beyond the ability of the individual to prevent, control, or direct them. Lastly, "abnormal" refers to movements that, either in kind or quantity, appeared outside the range of movements expected to occur within the context of a clinical interview.

Each occurrence of a spontaneous, involuntary, abnormal movement was classified into one of nine categories: chorea, athetosis, choreoathetosis, akathisia, ballismus, dystonia, resting tremor, intention tremor, and "other". Classification decisions were made using the following operational descriptions based on current neurology texts (e.g., Kaufmann, 2001). Chorea: quick, discrete, non-rhythmic, jerky movements, which tended to be abrupt in onset and seemed unpredictable in sequence and timing. Athetosis: slow, continuous, writhing movements, which appeared as a sequence of extension-flexion, pronation-supination movements. Choreoathetosis: slow, writhing movements punctuated by jerky movements within the same body region. Akathisia: repetitive, restless, fidgety movements. Ballismus: sudden, large, flinging or flailing movements. Dystonia: twisting movements that seemed to result from slow, sustained spasms of agonist and antagonist muscles. Tremor: a fine, rhythmic, repetitive, oscillating motion that occurred when posture was relaxed (resting tremor), or while the individual was in motion (intention tremor). As the classifications were based solely on observation, the emphasis was placed on determining whether the movement resembled the operational description of the 
movement category and appeared to be spontaneous, involuntary, and abnormal (e.g., a brief, non-rhythmic, jerky movement was considered "chorea-like" and classified as chorea). Thus, these categorizations were not intended as clinical diagnoses. Finally, to ensure that a movement abnormality occurrence that did not fit one of these categories was not missed, the "other" category was used if the rater was unable to determine which type of involuntary movement was observed.

In addition, the part of the body (arms, hands/fingers, head, face, or trunk) in which the movement occurred was specified as well as the side of the body on which the abnormality occurred (right and/or left side for arms and hands/fingers). Specifically, arm movement comprised any involuntary movement from the shoulder down that involved the arm, hand, and fingers as a unit. Hand/finger movement comprised any involuntary movement occurring at and/or below the wrist, including movement that involved the hand and fingers as a unit, or movement localized to one or more fingers.

The start and stop time of each movement, its maximum intensity, and a confidence rating were coded on a one-second time base. Intensity was coded as: 1 - mild, 2 - moderate, and 3 - severe; confidence of abnormality was coded as: 0.5 - questionable and 1 - definite. The determination of intensity was based on the amplitude of the movement; confidence of abnormality was based on how sure the rater was that the movement was both abnormal and involuntary within the context of the interview. Movements were not coded if they were clearly voluntary or purposeful (e.g., expressive gestures), intentional (e.g., playing with an object hair, pen, etc.; non-repetitively shifting position), completely or nearly completely occluded by an object or other body region, or were the result of a movement in another part of the body (e.g., trunk movement occurring because leg position was shifted). 
Behavior coding was performed using The Observer Video-Pro 5.0 (Noldus, 2003), a program specifically designed for the collection and analysis of observational data. Within this program, each body region was considered as a behavioral class, and each movement category was treated as an element within each of the behavioral classes. Severity and abnormality were each considered modifier classes, with 'mild', 'moderate', and 'severe' being elements of the severity class and 'questionable' and 'definite' being elements of the abnormality class. Duration was automatically calculated by the program from the start and stop times of each movement occurrence and was rounded to the nearest second.

Weighted and Aggregate Scores. To obtain a single index reflecting duration, severity, and abnormality of movement, a weighted score was calculated for each instance of a movement category occurring within each body region. This weighted score equaled the product of the duration of the movement (in seconds), the severity score $(1,2$, or 3$)$, and the abnormality score ( 0.5 or 1$)$ for the occurrence.

The weighted scores for each occurrence were then summed to yield aggregate weighted scores for each participant, resulting in a weighted score for each movement category and an overall total weighted score. The score for each movement category was calculated by summing the weighted scores occurring within that category across all of the body regions in which that movement occurred. The overall total was calculated by summing all weighted scores across all categories of movement and all body regions.

Inter-rater reliability. Prior to coding the participant interviews, 25 videotaped interviews of non-study schizophrenia patients were used for training and to ascertain initial inter-rater reliability. Following training, study inter-rater reliability of ratings was assessed using 30 study participants, randomly ordered across groups and stratified to ensure equal 
inclusion of participants from each group. The primary rater and a second trained rater separately coded the video segments from each of the 30 participants.

Intra-class correlations were calculated using a two-way mixed effects model. The intraclass correlations were: overall total 0.90 ; akathisia 0.91 ; chorea 0.74 ; choreoathetosis 0.61 ; and rest tremor 0.79 . Dystonia and intention tremor were only observed in two participants, and athetosis, ballismus, and 'other' were not coded for any participants, therefore intra-class correlations were not calculated for those categories.

\section{RESULTS}

\subsection{Sample Characteristics}

One hundred seventy-six participants met the inclusion criteria described above. These participants comprised 59 index probands, 39 index siblings, 39 control probands, and 39 control siblings. Of those participants, 139 individuals also had video available for analysis. (Video unavailability was typically due to participant refusal or camera unavailability at the time of the interview.) The videotaped participants consisted of 51 index probands, 33 index siblings, 38 control probands, and 17 control siblings. No significant differences were found between those with video vs. those without video in age, ethnic group, sex, years of education, or self-reported history of alcoholism or substance abuse. All subsequent analyses included only those individuals for whom video was available.

\subsubsection{Group clinical attributes}

Based on the RDC, the index probands comprised 33 individuals who had only schizophrenia episodes, 4 who had only schizoaffective episodes, and 14 who had both schizophrenia and schizoaffective episodes. In addition, 19 index probands also reported a history of alcohol abuse and 20 also reported a history of substance abuse. The clinical characteristics of the index probands were as follows: age at first hospitalization (mean 24.14, 
standard deviation [4.8]), total months hospitalized (5.16 [4.4]), number of months since most recent hospitalization (37.9 [29.9]), percentage taking antipsychotic medication at the time of interview (98.0\%), total chlorpromazine $(\mathrm{mg})$ dosage equivalent (378.5 [361.0]), percentage taking antiparkinsonian medications (64.7\%), and total cogentin (mg) dosage equivalent (1.31 $[1.4])$.

The index siblings comprised two individuals who were diagnosed with a schizophrenia spectrum disorder, 16 who were included in the 'other' category (including four individuals who reported a history of alcohol abuse and six who reported a history of substance abuse), and 15 who were categorized as 'non-diagnosis'. The control probands included five individuals who were placed in the 'other' category (miscellaneous non-schizotypal, non-schizoid personality disorders) and 33 who were categorized as 'non-diagnosis'; consistent with the study design, none of the control probands reported a history of alcoholism or substance abuse. The control siblings consisted of eight individuals who were included in the 'other' category (including one individual who reported history of alcohol abuse and two who reported a history of substance abuse) and nine who were categorized as 'non-diagnosis'.

\subsubsection{Group demographic comparisons}

Table 1 presents the demographic characteristics of the participant groups. Groups were compared on the variables of age, sex, ethnicity, handedness, years of education, and alcohol or substance abuse in order to identify potential covariates. Planned comparisons (2-tailed) indicated that the control probands and control siblings did not significantly differ on any of the variables assessed except, as expected, lifetime alcohol or substance abuse $\left(\mathrm{X}^{2}=7.093, d f=1, \mathrm{p}\right.$ $=.008)$. Therefore, in order to increase sample size, the control proband and control sibling groups were combined in subsequent demographic comparisons. 
The index proband group was significantly older $(\mathrm{t}=2.91, d f=104, \mathrm{p}=0.004)$, consisted of more males $\left(\mathrm{X}^{2}=10.17, d f=1, \mathrm{p}=0.001\right)$ and fewer European Americans $\left(\mathrm{X}^{2}=4.45, d f=\right.$ $1, \mathrm{p}=0.035)$, and endorsed more lifetime alcohol and substance abuse $\left(\mathrm{X}^{2}=25.84, d f=1, \mathrm{p}=\right.$ 0.001) than the combined control group. In addition, the index proband group consisted of significantly more males $\left(\mathrm{X}^{2}=9.14, d f=1, \mathrm{p}=0.002\right)$ and endorsed significantly more lifetime alcohol and substance abuse than the index sibling group $\left(\mathrm{X}^{2}=5.16, d f=1, \mathrm{p}=0.023\right)$. Lastly, in the index sibling group, there was significantly more lifetime alcohol and substance abuse $\left(\mathrm{X}^{2}\right.$ $=6.66, d f=1, \mathrm{p}=0.010)$ and a trend towards significantly fewer European Americans $\left(\mathrm{X}^{2}=\right.$ 3.66, $d f=1, \mathrm{p}=0.056)$ than the combined controls. No other demographic comparisons were significant.

Table 1: Demographic Characteristics

\begin{tabular}{|c|c|c|c|c|c|}
\hline Demographic characteristic & $\begin{array}{l}\text { Index probands } \\
(\mathrm{n}=51)\end{array}$ & $\begin{array}{l}\text { Index siblings } \\
(\mathrm{n}=33)\end{array}$ & $\begin{array}{l}\text { Combined controls } \\
(n=55)\end{array}$ & $\begin{array}{l}\text { Control probands } \\
(n=38)\end{array}$ & $\begin{array}{l}\text { Control siblings } \\
(\mathrm{n}=17)\end{array}$ \\
\hline $\begin{array}{l}\text { Age } \\
\text { years; mean (SD) }\end{array}$ & $33.0(6.9)$ & $30.8(7.1)$ & $29.3(6.3)$ & $28.8(5.9)$ & $30.2(7.1)$ \\
\hline $\begin{array}{l}\text { Sex } \\
\text { male; } \mathrm{n}(\%)\end{array}$ & $37(72.5)$ & $13(39.4)$ & $23(41.8)$ & $17(44.7)$ & $6(35.3)$ \\
\hline $\begin{array}{l}\text { Ethnic Group }{ }^{1} \\
\text { European American; } n \text { (\%) }\end{array}$ & $40(78.4)$ & $26(78.8)$ & $51(92.7)$ & $35(92.1)$ & $16(94.1)$ \\
\hline $\begin{array}{l}\text { Handedness }{ }^{2} \\
\text { right; } \mathrm{n}(\%)\end{array}$ & $42(85.7)$ & $25(80.6)$ & $46(83.6)$ & $31(81.6)$ & $15(88.2)$ \\
\hline $\begin{array}{l}\text { Education } \\
\text { total years; mean (SD) }\end{array}$ & $13.2(2.4)$ & $13.9(3.1)$ & $14.1(2.5)$ & $14.1(2.7)$ & $14.0(1.8)$ \\
\hline $\begin{array}{l}\text { Alcohol and/or substance abuse } \\
\mathrm{n}(\%)\end{array}$ & $25(49.0)$ & $8(24.2)$ & $3(5.5)$ & $0(0.0)$ & 3 (17.6) \\
\hline
\end{tabular}

1 - European American or African American

2 - right, left, or ambidextrous 


\subsection{Spontaneous Dyskinesia}

\subsubsection{Preliminary analyses}

Initial inspection of the aggregate variables determined that all outliers were valid and hence were included in all subsequent analyses. The distributions of all aggregate variables were highly positively skewed in the total group. (Skewness ranged from 2.86 to 10.10 , standard error 0.20.) Due to the non-normal distribution of these variables, non-parametric tests were used on all analyses and all tests were one-tailed $(\mathrm{p} \leq 0.05)$ due to directional hypotheses. Specifically, logistic regression, a non-parametric technique that does not rely on assumptions of normality for

predicting variables, was used (Hosmer \& Lemeshow, 1989). In this case, the measures of spontaneous dyskinesia were utilized to predict group membership (e.g., schizophrenia vs. controls). No occurrences of the movement categories ballismus and other were noted, thus these categories were not included in analyses. The means and standard deviations of the aggregate variables are presented in Table 2. 


\section{Table 2: Spontaneous Dyskinesia}

\begin{tabular}{|c|c|c|c|}
\hline Aggregate variable $^{1}$ & $\begin{array}{l}\text { Index probands } \\
(\mathrm{n}=51)\end{array}$ & $\begin{array}{l}\text { Index siblings } \\
(\mathrm{n}=33)\end{array}$ & $\begin{array}{l}\text { Combined controls } \\
(n=55)\end{array}$ \\
\hline Overall Total & $732.2(836.5)$ & $291.9(404.8)$ & $216.2(291.5)$ \\
\hline Chorea & $42.2(78.2)$ & $29.8(39.5)$ & $22.8(40.6)$ \\
\hline Athetosis & $0.8(5.3)$ & $0.0(0.0)$ & $0.2(1.7)$ \\
\hline Choreoathetosis & $4.6(26.6)$ & $0.7(2.7)$ & $1.1(3.9)$ \\
\hline Akathesia & $658.0(838.9)$ & $255.3(405.3)$ & $191.6(286.5)$ \\
\hline Right Arm & $3.1(21.0)$ & $0.0(0.0)$ & $0.0(0.0)$ \\
\hline Right Hand \& Fingers & 320.9 (455.9) & $115.2(194.6)$ & $91.6(147.1)$ \\
\hline Left Arm & $7.7(36.7)$ & $0.0(0.0)$ & $0.1(0.41)$ \\
\hline Left Hand \& Fingers & $313.8(411.6)$ & $140.1(236.9)$ & $99.1(157.6)$ \\
\hline Head & $0.7(4.2)$ & $0.0(0.0)$ & $0.1(0.3)$ \\
\hline Face & $0.0(0.0)$ & $0.0(0.0)$ & $0.0(0.0)$ \\
\hline Trunk & $11.8(62.7)$ & $0.0(0.0)$ & $0.9(3.7)$ \\
\hline Dystonia & $0.9(3.3)$ & $0.38(1.4)$ & $0.6(3.1)$ \\
\hline Rest Tremor & $17.6(95.4)$ & $0.3(1.4)$ & $0.0(0.0)$ \\
\hline Right Arm & $0.0(0.0)$ & $0.0(0.0)$ & $0.0(0.0)$ \\
\hline Right Hand \& Fingers & $12.0(80.7)$ & $0.0(0.0)$ & $0.0(0.0)$ \\
\hline Left Arm & $0.23(1.4)$ & $0.0(0.0)$ & $0.0(0.0)$ \\
\hline Left Hand \& Fingers & $5.3(28.1)$ & $0.33(1.4)$ & $0.0(0.0)$ \\
\hline Head & $0.0(0.0)$ & $0.0(0.0)$ & $0.0(0.0)$ \\
\hline Face & $0.0(0.0)$ & $0.0(0.0)$ & $0.0(0.0)$ \\
\hline Trunk & $0.0(0.0)$ & $0.0(0.0)$ & $0.0(0.0)$ \\
\hline Intention Tremor & $8.3(33.9)$ & $5.5(31.5)$ & $0.0(0.0)$ \\
\hline Right Arm & $0.8(5.5)$ & $0.0(0.0)$ & $0.0(0.0)$ \\
\hline Right Hand \& Fingers & $4.6(20.8)$ & $2.8(15.8)$ & $0.0(0.0)$ \\
\hline Left Arm & $0.2(1.1)$ & $0.0(0.0)$ & $0.0(0.0)$ \\
\hline Left Hand \& Fingers & $1.7(11.8)$ & $2.7(15.7)$ & $0.0(0.0)$ \\
\hline Head & $1.1(7.6)$ & $0.0(0.0)$ & $0.0(0.0)$ \\
\hline Face & $0.0(0.0)$ & $0.0(0.0)$ & $0.0(0.0)$ \\
\hline Trunk & $0.0(0.0)$ & $0.0(0.0)$ & $0.0(0.0)$ \\
\hline
\end{tabular}

${ }^{1}$ weighted score, mean (SD)

Internal consistency. Cronbach's alpha was 0.01 for the total weighted score within the total sample, indicating that the seven movement categories were uncorrelated. In addition, Cronbach's alpha was calculated for each of the movement categories (with the seven body regions as items): athetosis (0.73), chorea (0.26), choreoathetosis (0.01), akathisia $(0.56)$, 
dystonia (0.06), rest tremor (0.31), and intention tremor (0.57). It is not surprising that movement occurrences in one category would not necessarily be associated with occurrences of other categories of movement in the same individual, or that within a category, movement in one body region would not be associated with other regions. Given this, it is interesting that for some of the above categories, movement occurrences seem to be moderately correlated across body regions.

Control probands vs. control siblings. Next, control probands and control siblings were compared using logistic regression analysis. The control proband and control sibling groups did not significantly differ $(p \geq 0.257)$ on any of the aggregate variables and therefore were combined for all subsequent analyses.

\subsubsection{Index probands vs. controls}

The index proband group and the combined control group were compared using logistic regression analysis with age, sex, ethnic group, and alcohol and substance abuse as covariates. As can be seen in Table 3, the index probands had a significantly higher overall total involuntary movement score compared to the combined controls. Based on the total score, $82 \%$ of the individuals in the index proband group were classified as probands and $84 \%$ of those in the combined control group were classified as controls (see Appendix A). The index proband group also had a significantly higher score on the individual movement categories of akathisia, rest tremor, and intention tremor. Equivalent results were found when the index proband group was compared separately with the control proband and control sibling groups with one minor exception: intention tremor was not significant when index probands were compared with only the control sibling group. Using a Bonferroni correction for the seven movement category comparisons, akathisia and rest tremor remained significant. No significant interactions were 
found with age or sex for either the overall total or any of the movement categories. Among index probands, neither total chlorpromazine dose equivalent nor cogentin dose equivalent correlated with the scores for any of the movement categories (range: $r=-0.17-0.19$ ).

Additional pairwise analyses were completed to investigate the contribution of each movement category, after controlling for all other categories. Specifically, after all covariates (listed above) were entered into the logistic regression equation, all movement categories except one were entered together in one step. The remaining category was entered in the final step to determine if it significantly differentiated the participant groups over and above the other categories. This procedure was completed for each movement category; only akathisia remained significantly higher in the index proband group compared to the combined control group $(\mathrm{p}=$ 0.001) after controlling for the other movement categories.

To investigate the significant findings for akathisia, rest tremor, and intention tremor, exploratory analyses were conducted to compare the index proband group and the combined control group on the body regions in which these movement categories occurred. Age, sex, ethnic group, and alcohol and substance abuse were again used as covariates; the results are presented in Table 3. For akathisia, the index proband group had a significantly higher score for the regions of right arm, right hand/fingers, left arm, left hand/fingers, and trunk. A significant interaction between age and right hand/fingers was observed $(p=0.018)$. Examination of the data suggested that this interaction was driven by the results from only a few participants and was not indicative of a trend in the sample as a whole. Nevertheless, the analysis of akathisia within the region of right hand/fingers was rerun without age as a covariate, and the findings for this region remained significant. After Bonferroni correction for the seven body regions, right hand/fingers and left hand/fingers remained significant. 
Regarding rest tremor, the index proband group had a significantly higher score than the combined control group for the body regions of right hand/fingers, left arm, and left hand/fingers. Only right hand/fingers remained significant after Bonferroni correction. For intention tremor, the index probands had a significantly higher score than the combined controls for the regions of right arm, right hand/fingers, and head. None of the body regions remained significant following correction using the Bonferroni method. No additional interactions were found with age or sex for any of the body regions.

\subsubsection{Index probands vs. index siblings}

The index proband group and the index sibling group were then compared using logistic regression; sex and alcohol and substance abuse were used as covariates. As presented in Table 3 , the index probands had a significantly higher overall total score when compared to the index sibling group. Additionally, on the basis of the total score $84 \%$ of the individuals in the index proband group were categorized as probands and $52 \%$ of those in the index sibling group were categorized as siblings (see Appendix A). It is interesting to note that $48 \%$ of the siblings were categorized as probands based on the total scores of the individuals in these two groups. When each category was assessed separately, the index proband group had a significantly higher score on akathisia and rest tremor. Akathisia remained significant after Bonferroni correction. In addition, after controlling for all other movement categories using the method described above, akathisia and rest tremor both remained significantly higher in the index probands compared to the index siblings $(\mathrm{p}=.002$ and $\mathrm{p}=.033$, respectively). No interactions with age or sex were found for either the overall total or the movement categories.

To further explore the findings for akathisia and rest tremor, the index proband group and the index sibling group were compared on the body regions in which these movements were 
observed. Sex and alcohol and substance abuse were again entered as covariates. As shown in Table 3, for akathisia, the index proband group had a significantly higher score for the regions of right arm, right hand/fingers, left arm, left hand/fingers, and trunk. Right hand/fingers, left arm, and left hand/fingers remained significant following Bonferroni correction. For rest tremor, the index proband group had a significantly higher score for right hand/fingers and a trend towards having a significantly higher score for left arm. After applying the Bonferroni method, right hand/fingers remained significant. No interactions were found with age or sex for any of the body regions.

\subsubsection{Index siblings vs. controls}

Most importantly, the index sibling group and the combined control group were compared next using logistic regression analysis. Ethnic group and alcohol and substance abuse were used as covariates. As presented in Table 3, the index sibling group did not differ significantly from the combined controls on the overall total score. In addition, based on the total score $87 \%$ of the individuals in the combined control group were classified as controls and $39 \%$ of individuals in the index sibling group were classified as siblings (see Appendix A). Thus in this comparison, $61 \%$ of the siblings were classified as controls. The index sibling group did have a significantly higher score than the combined control group on the movement category of rest tremor and a trend towards significance for the category of intention tremor, although neither was significant following Bonferroni correction. When compared with each control group separately, the index sibling group did not differ significantly on any movement categories from the control probands and only showed a trend toward significance on rest tremor $(\mathrm{p}=0.07)$ compared to the control siblings. Additionally, when all other movement categories were controlled for, only the score for rest tremor approached significance $(\mathrm{p}=.058)$; intention tremor 
was not significant. It should be noted that the mean scores for rest and intention tremor were low in the index sibling group and were zero in the control group (see Table 2). No interactions were found with age or sex for either the overall total or the movement categories.

To investigate the findings for rest and intention tremor, the index sibling group and the combined control group were compared on the relevant body regions, with ethnic group and alcohol and substance abuse entered as covariates. As can be seen in Table 3, for rest tremor, the index sibling group had a significantly higher score for the body region of left hand/fingers. For intention tremor, the index sibling group showed a trend towards significance for the regions of right hand/fingers and left hand/fingers. None of the regions remained significant for either rest or intention tremor following Bonferroni correction. No interactions were found with age or sex for any of the body regions. 
Table 3: Pairwise Group Analyses: Overall Total and Movement Categories

\begin{tabular}{|c|c|c|c|}
\hline Aggregate variable & $\begin{array}{l}\text { Index probands vs. } \\
\text { Combined controls }\end{array}$ & $\begin{array}{l}\text { Index probands vs. } \\
\text { Index siblings }{ }^{2}\end{array}$ & $\begin{array}{l}\text { Index siblings vs. } \\
\text { Combined controls }\end{array}$ \\
\hline Overall Total & 0.001 & 0.001 & 0.227 \\
\hline Chorea & 0.289 & 0.374 & 0.424 \\
\hline Athetosis & 0.440 & 0.273 & 0.203 \\
\hline Choreoathetosis & 0.192 & 0.252 & 0.309 \\
\hline Akathesia & $0.001^{\dagger}$ & $0.002^{\dagger}$ & 0.259 \\
\hline Right Arm & 0.014 & 0.023 & na \\
\hline Right Hand \& Fingers & $0.001^{\dagger}$ & $0.002^{\dagger}$ & na \\
\hline Left Arm & 0.050 & $0.006^{\dagger}$ & na \\
\hline Left Hand \& Fingers & $0.002^{\dagger}$ & $0.007^{\dagger}$ & na \\
\hline Head & 0.310 & 0.135 & na \\
\hline Face & - & - & - \\
\hline Trunk & 0.019 & 0.014 & na \\
\hline Dystonia & 0.203 & 0.225 & 0.423 \\
\hline Rest Tremor & $0.002^{\dagger}$ & 0.021 & 0.012 \\
\hline Right Arm & - & - & - \\
\hline Right Hand \& Fingers & $0.003^{\dagger}$ & $0.006^{\dagger}$ & - \\
\hline Left Arm & 0.021 & 0.055 & - \\
\hline Left Hand \& Fingers & 0.020 & 0.071 & 0.012 \\
\hline Head & - & - & - \\
\hline Face & - & - & - \\
\hline Trunk & - & - & - \\
\hline Intention Tremor & 0.013 & 0.183 & 0.057 \\
\hline Right Arm & 0.023 & na & - \\
\hline Right Hand \& Fingers & 0.014 & na & 0.057 \\
\hline Left Arm & 0.372 & na & - \\
\hline Left Hand \& Fingers & 0.401 & na & 0.057 \\
\hline Head & 0.023 & na & - \\
\hline Face & - & - & - \\
\hline Trunk & - & - & - \\
\hline
\end{tabular}

Notes: p-value obtained from pairwise logistic regression analysis, 1-tailed;

1 covariates: age, sex, ethnic group, alcohol and substance abuse

2 covariates: sex, alcohol and substance abuse

${ }^{3}$ covariates: ethnic group, alcohol and substance abuse

${ }^{\dagger}$ comparisons that remained significant after applying Bonferroni correction for 7 comparisons $(p<0.007)$

- not calculated (no assessments of the movement category made in this region)

na - not applicable (overall movement category not significant) 


\subsubsection{Sibling Correlations}

Sibling correlations (Pearson Product-Moment) were calculated for the overall total and each of the movement categories. Control probands and their siblings $(n=16$ pairs) were not significantly correlated on either the overall total $(\mathrm{r}=-0.08, \mathrm{p}=0.385)$ or any of the movement categories (range: $\mathrm{r}=-0.12--0.05, \mathrm{p}>0.325$ ). Furthermore, index probands and their siblings $(n=29$ pairs $)$ also were not significantly correlated on the overall total $(r=0.05, p=0.402)$ or the movement categories (range: $\mathrm{r}=-0.11-0.24, \mathrm{p}>0.105$ ).

\section{DISCUSSION}

The results of this study indicated that stable, medicated, schizophrenia or schizoaffective outpatients displayed significantly more spontaneous, involuntary movement abnormalities compared to controls. Specifically, akathisia, rest tremor, and intention tremor were significantly elevated in this patient sample. These schizophrenia outpatients also displayed significantly more involuntary movements (akathisia and rest tremor) compared to their non-psychotic siblings. Notably, siblings of schizophrenia patients did not display significantly more involuntary movement abnormalities compared to controls, although rest and intention tremor were observed in a few individuals.

\subsection{Comparison with previous studies}

As stated above, schizophrenia probands displayed significantly more spontaneous dyskinesia compared to controls. In patients, akathisia was observed to occur more frequently in the right and left arms, right and left hands/fingers, and trunk. Further, rest tremor was significantly elevated in the left arm and left and right hands/fingers, and intention tremor was significantly elevated in the right arm, right hands/fingers, and head. Of note, rest and intention tremor were absent in the control group. 
The finding that individuals with schizophrenia display more involuntary movements than controls is consistent with results from previous studies of medicated and unmedicated schizophrenia patients (Friedman, 2004). Previous observations of spontaneous dyskinesia in individuals with schizophrenia who are neuroleptic naïve suggest that although medication certainly may be contributory, it is not the only cause of such movements (Fenton, 2000; McCreadie et al., 1996). Also, although not a definitive measure of the influence of medication, in the current patient sample neither total chlorpromazine dose equivalent nor cogentin dose equivalent correlated significantly with the scores for any of the movement categories. Interestingly, the specific types of involuntary movements observed in previous samples of neuroleptic naïve patients included choreoathetoid movements, tremor, akathisia, and dystonia, all of which were observed in the current sample of schizophrenia patients. Importantly, the current finding of a significant elevation in spontaneous dyskinesia in patients compared to controls, a finding that is consistent with previous reports, further supports that the method employed in this investigation is sensitive to detecting spontaneous, involuntary movement abnormalities.

Schizophrenia index probands also displayed significantly more involuntary movements compared to their non-psychotic siblings. Compared to siblings, akathisia in patients was again observed more frequently in the right and left arms, right and left hands/fingers, and trunk. In contrast to the comparison with controls, rest tremor was only observed more frequently in the right hands/fingers, with a trend toward significance in the left arm.

Only a few prior studies have compared spontaneous involuntary movement abnormalities occurring in schizophrenia patients and their relatives. The majority of studies included medicated schizophrenia patients and reported that these patients displayed more 
involuntary movements than their relatives (Egan et al., 2001; Ismail et al., 1998; Yazici et al., 2002). These findings are congruent with the results of the current study. In addition, one study was located that compared neuroleptic naïve schizophrenia patients and their siblings (McCreadie et al., 2003). In that study, significantly more neuroleptic naïve patients had at least mild dyskinesia (33\%) compared to their non-psychotic siblings (16\%).

Despite the elevations of spontaneous dyskinesia among the current sample of patients noted above, the index sibling group did not display significantly more involuntary movement abnormalities compared to controls when all movement categories were summed together. However, when each category was explored separately, a significant elevation of rest tremor $(\mathrm{p}=$ $0.012)$ and a trend toward significance in intention tremor $(p=0.057)$ emerged. These elevations were not significant when a Bonferroni correction was applied. Upon further analysis, rest tremor was present in only two siblings and intention tremor was observed in only one sibling, but neither was present in controls. In these siblings, rest tremor occurred in the left hand/fingers and intention tremor occurred in the left and right hands/fingers. It is possible that tremor is a noteworthy difference between siblings and controls but that our sample sizes were too small to adequately detect this effect. Alternatively, such isolated results could reflect false positive statistical errors. Overall, based on our results, we conclude that it is unlikely that major differences exist in spontaneous dyskinesia between siblings and controls.

Previous findings regarding the occurrence of spontaneous dyskinesia in first-degree relatives of individuals with schizophrenia are sparse and inconclusive. As reviewed above, four studies (Chen et al., 2000; Flyckt et al., 1999; Kinney et al., 1986; McCreadie et al., 1996) have specifically reported on the percentage of spontaneous involuntary movements in parents or siblings of schizophrenia patients compared with controls. Two of the four studies reported a 
higher percentage of spontaneous dyskinesia in relatives (Flyckt et al., 1999; Kinney et al., 1986), and two reported no difference (Chen et al., 2000; McCreadie et al., 1996). The results of the current study are consistent with the latter, negative findings.

Several factors could contribute to this discrepancy. For example, the studies with positive findings included relatives who were older than controls. Also, it appears that these studies had more liberal inclusion criteria for relatives than for controls, which could increase the possibility of false positive results. For instance, Kinney et al. (1986) excluded relatives diagnosed with schizophrenia and Flyckt et al. (1999) excluded relatives with a psychotic disorder, but both excluded controls with any psychiatric disorder. In contrast, Chen et al. (2000), who found no significant differences, excluded both relatives and controls with any psychiatric illness.

\subsection{Strengths and limitations of current study}

This study sought to investigate the presence of involuntary movements in individuals with schizophrenia and their siblings while minimizing several of the inconsistencies and weaknesses present in the literature to date. Specifically, four areas were addressed: assessment methodology, rater blindness, inter-rater reliability, and demographic matching of participants.

Use of videotaped assessments conferred several advantages, including: allowing for "informal" observation of involuntary movements, permitting detailed assessment of these movements, facilitating the use of blinded ratings, and allowing for additional ratings to ensure reliability. According to the current neurology literature, spontaneous involuntary movements can be made worse by the stress of a formal neurological exam (Cadet et al., 1986). This potential source of stress was avoided by observing (on videotape) whether these abnormalities occurred spontaneously during the administration of a standardized, clinical assessment 
interview. Each individual was carefully observed for the presence of several types of abnormal, involuntary movements. These movements were additionally coded to obtain information on abnormality and severity of the movement. This coding scheme, in addition to the use of digital media and the Observer Pro computerized rating program, facilitated a much more thorough evaluation of spontaneous dyskinesia that is possible in the typical neurological exam. Furthermore, all ratings were conducted blind to group status by a single individual who obtained good reliability with a second individual who also was trained on this coding system.

Attempts to match participants on age, sex, ethnic group, and education were for the most part successful, with a few exceptions. Most importantly, the index sibling group was not significantly different on demographics compared to the combined control group. However, the index proband group was older, consisted of more males, and fewer European Americans than the combined control group, and had more males than the index sibling group. These differences were controlled statistically during pairwise comparisons.

In addition, two control groups were included to address potential concerns that recruitment strategies may result in a "super-normal" group that was not representative of the general population. By including siblings of controls who are subject to fewer exclusion criteria, we hoped to obtain a more representative control group. As presented earlier, the control groups did not differ and thus were combined in subsequent analyses to increase power. Furthermore, when index probands and index siblings were compared with each control group separately, the results were largely equivalent.

Potential methodological constraints of the current study also should be addressed. First, only movement abnormalities affecting the upper limbs, head, face, and trunk of each participant were assessed. Thus, any involuntary movements occurring in the lower limbs would have been 
missed. In previous studies, spontaneous dyskinesia observed in schizophrenia patients, relatives, and schizotypal personality disorder (SPD) individuals most often occurred in the upper body (e.g., Walker et al., 1999), however it cannot be ruled out that inclusion of lower limbs in our assessment might have altered our results.

Second, a measure of anxiety was not included in this study. It is possible that group differences in anxiety level during the interview may account for a portion of the differences in involuntary movement scores. For example, to the extent that anxiety may worsen involuntary movements in general (Cadet et al., 1986) and that the index probands were more anxious during the interview than other participants, heightened anxiety in index probands might have contributed to a greater incidence of involuntary movements in this group, potentially inflating differences between the index probands and the other groups.

Third, as noted above, it is also possible that the current study did not have sufficient power to detect significant differences with a small effect size. Power calculations indicated that our sample sizes (harmonic mean $=41$ group when control groups are combined) allowed detection of effect sizes between index siblings and controls of 0.80 (large effect) with power of 0.97 and effect sizes of 0.50 (medium effect) with power of 0.72 . However, this study was underpowered (0.22) to detect an effect size of 0.20 (small effect) $(\mathrm{p} \leq 0.05$, one-tailed) (Cohen, 1987). Thus, a small effect between index siblings and controls probably would not have been detected in this study.

\subsection{Implications}

Spontaneous dyskinesia was more frequently observed in individuals with schizophrenia than controls. However, the lack of significant differences between siblings and controls argues against an association between spontaneous dyskinesia and an underlying genetic liability to 
schizophrenia. Thus, in light of the results of this study, it appears that involuntary movement abnormalities may be limited in their utility as endophenotypes in genetic studies of schizophrenia.

The significant elevation in spontaneous dyskinesia in patients compared to nonpsychotic relatives and controls suggests that some involuntary movement abnormalities may be due to genetic variation or environmental experiences that are unique to patients. In particular, akathisia most significantly distinguished index probands from controls and index siblings, yet did not significantly differentiate the siblings from controls. It is unclear what genetic or environmental effects, beyond medication, may lead to spontaneous dyskinesia in schizophrenia patients. Additional investigation along these lines may be informative regarding the etiological agents that produce psychosis in some at risk individuals, but not in others. 


\section{APPENDIX A}

\section{Classification Tables Based on Overall Total}

$\underline{\text { Index Probands vs. Combined Controls }}$

\begin{tabular}{lllllc}
\hline Observed group & \multicolumn{3}{l}{ Predicted Group } \\
& \multicolumn{3}{l}{ Index Probands } & Combined Controls \\
\hline & Observed n & Predicted n & $\%$ of group & Predicted n & $\%$ of group \\
\hline Index Probands & 51 & 42 & $82 \%$ & 9 & $18 \%$ \\
\hline Combined Controls & 55 & 9 & $16 \%$ & 46 & $84 \%$ \\
\hline
\end{tabular}

$\underline{\text { Index Probands vs. Index Siblings }}$

\begin{tabular}{llllll}
\hline Observed group & \multicolumn{2}{l}{ Predicted Group } \\
& & \multicolumn{2}{l}{ Index Probands } & Index Siblings \\
\hline & Observed n & Predicted n & $\%$ of group & Predicted n & $\%$ of group \\
\hline Index Probands & 51 & 43 & $84 \%$ & 8 & $16 \%$ \\
\hline Index Siblings & 33 & 16 & $48 \%$ & 17 & $52 \%$ \\
\hline
\end{tabular}

$\underline{\text { Index Siblings vs. Combined Controls }}$

\begin{tabular}{lllllc}
\hline Observed group & \multicolumn{2}{l}{ Predicted Group } \\
\hline & \multicolumn{2}{l}{ Index Siblings } & Combined Controls \\
\hline & Observed n & Predicted n & $\%$ of group & Predicted n & $\%$ of group \\
\hline Index Siblings & 33 & 13 & $39 \%$ & 20 & $61 \%$ \\
\hline Combined Controls & 55 & 7 & $13 \%$ & 48 & $87 \%$
\end{tabular}




\section{REFERENCES}

Antonini, A., Moeller, J. R., Nakamura, T., Spetsieris, P., Dhawan, V., \& Eidelberg, D. (1998). The metabolic anatomy of tremor in Parkinson's disease. Neurology, 51(3), 803-810.

Cadet, J. L., Rickler, K. C., \& Weinberger, D. R. (1986). The clinical neurologic examination in schizophrenia. In H. A. Nasrallah \& D. R. Weinberger (Eds.), The Neurology of Schizophrenia (Vol. 1). Amsterdam: Elsevier Science Publishers B.V.

Caligiuri, M. P., Lohr, J. B., \& Jeste, D. V. (1993). Parkinsonism in neuroleptic-naive schizophrenic patients. American Journal of Psychiatry, 150(9), 1343-1348.

Cardno, A. G., Marshall, E. J., Coid, B., Macdonald, A. M., Ribchester, T. R., Davies, N. J., et al. (1999). Heritability estimates for psychotic disorders. Archives of General Psychiatry, $56,162-168$.

Cassady, S., Adami, H., Moran, M., Kunkel, R., \& Thaker, G. K. (1998). Spontaneous dyskinesias in subjects with schizophrenia spectrum personality disorders. American Journal of Psychiatry, 155, 70-75.

Chatterjee, A., Chakos, M., Koreen, A., Geisler, S., Sheitman, B., Woerner, M., et al. (1995). Prevalence and clinical correlates of extrapyramidal signs and spontaneous dyskinesia in never-medicated schizophrenic patients. The American Journal of Psychiatry, 152(12), 1724-1729.

Chen, R., Chen, E., \& Mak, F. (2000). Soft neurological signs in schizophrenic patients and their nonpsychotic siblings. Journal of Nervous \& Mental Disease, 188, 84-89.

Chorfi, M., \& Moussaoui, D. (1989). Lack of dyskinesias in unmedicated schizophrenics. Psychopharmacology, 97, 423. 
Cohen, J. (1987). Statistical Power Analysis for the Behavioral Sciences (2nd ed.). Hillsdale, N.J.: Lawrence Earlbaum.

Corson, P. W., O'Leary, D. S., Miller del, D., \& Andreasen, N. C. (2002). The effects of neuroleptic medications on basal ganglia blood flow in schizophreniform disorders: A comparison between the neuroleptic-naive and medicated states. Biological Psychiatry, 52(9), 855-862.

Crane, G. E. (1968). Tardive dyskinesia in patients treated with major neuroleptics: A review of the literature. American Journal of Psychiatry, 124(February supplement), 40-48.

Craver, J. C., \& Pogue-Geile, M. F. (1999). Familial liability to schizophrenia: a sibling study of negative symptoms. Schizophrenia Bulletin, 25(4), 827-839.

Danos, P., Baumann, B., Bernstein, H. G., Stauch, R., Krell, D., Falkai, P., et al. (2002). The ventral lateral posterior nucleus of the thalamus in schizophrenia: a post-mortem study. Psychiatry Research, 114(1), 1-9.

Dooling, E. C., \& Adams, R. D. (1975). The pathological anatomy of posthemiplegic athetosis. Brain, 98, 29-48.

Egan, M. F., Hyde, T. M., Bonomo, J. B., Mattay, V. S., Bigelow, L. B., Goldberg, T. E., et al. (2001). Relative risk of neurological signs in siblings of patients with schizophrenia. American Journal of Psychiatry, 158, 1827-1834.

Endicott, J., \& Spitzer, R. L. (1978). A diagnostic interview: The Schedule for Affective Disorders and Schizophrenia. Archives of General Psychiatry, 35, 837-844.

Fenn, D. S., Moussaoui, D., Hoffman, W. F., Kadri, N., Bentounssi, B., Tilane, A., et al. (1996). Movements in never-medicated schizophrenics: a preliminary study. Psychopharmacology, 123, 206-210. 
Fenton, W. (2000). Prevalence of spontaneous dyskinesia in schizophrenia. Journal of Clinical Psychiatry, 61 Suppl 4, 10-14.

Flyckt, L., Sydow, O., Bjerkenstedt, L., Edman, G., Rydin, E., \& Wiesel, F.-A. (1999).

Neurological signs and psychomotor performance in patients with schizophrenia, their relatives and healthy controls. Psychiatry Research, 86, 113-129.

Friedman, J. H. (2004). Historical perspectives on movement disorders. Journal of Clinical Psychiatry, 65 Suppl 9, 3-8.

Gervin, M., Browne, S., Lane, A., Clarke, M., Waddington, J. L., Larkin, C., et al. (1998). Spontaneous abnormal involuntary movements in first-episode schizophrenia and schizophreniform disorder: baseline rate in a group of patients from an Irish catchment area. American Journal of Psychiatry, 155(9), 1202-1206.

Gil-ad, I., Shtaif, B., Shiloh, R., \& Weizman, A. (2001). Evaluation of the neurotoxic activity of typical and atypical neuroleptics: relevance to iatrogenic extrapyramidal symptoms. Cellular and Molecular Neurobiology, 21(6), 705-716.

Gottesman, I. I., \& Gould, T. D. (2003). The endophenotype concept in psychiatry: etymology and strategic intentions. American Journal of Psychiatry, 160, 636-645.

Gottesman, I. I., \& Shields, J. (1972). Schizophrenia and Genetics: A Twin Study Vantage Point. New York, NY: Academic Press.

Gunduz, H., Wu, H., Ashtari, M., Bogerts, B., Crandall, D., Robinson, D. G., et al. (2002). Basal ganglia volumes in first-episode schizophrenia and healthy comparison subjects. Biological Psychiatry, 51(10), 801-808.

Hosmer, D. W., \& Lemeshow, S. (1989). Applied Logistic Regression. New York, NY: John Wiley \& Sons. 
Ismail, B., Cantor-Graae, E., \& McNeil, T. F. (1998). Neurological abnormalities in schizophrenic patients and their siblings. American Journal of Psychiatry, 155(1), 84-89.

Jernigan, T. L., Zisook, S., Heaton, R. K., Moranville, J. T., Hesselink, J. R., \& Braff, D. L. (1991). Magnetic resonance imaging abnormalities in lenticular nuclei and cerebral cortex in schizophrenia. Archives of General Psychiatry, 48, 881-890.

Kaufmann, D. (2001). Clinical Neurology for Psychiatrists (5th ed.). Philadelphia, PA: W.B. Saunders Company.

Keshavan, M. S., Rosenberg, D., Sweeney, J. A., \& Pettegrew, J. W. (1998). Decreased caudate volume in neuroleptic-naive psychotic patients. American Journal of Psychiatry, 155(6), 774-778.

Kim, J.-S., Lee, K.-S., Lee, K.-H., Kim, Y.-I., Kim, B.-S., Chung, Y.-A., et al. (2002). Evidence of thalamic disinhibition in patients with hemichorea: semiquantitative analysis using SPECT. Journal of Neurology, Neurosurgery and Psychiatry, 72(3), 329-333.

Kinney, D. K., Woods, B. T., \& Yurgelun-Todd, D. M. (1986). Neurological abnormalities in schizophrenic patients and their families: II. Neurologic and psychiatric findings in relatives. Archives of General Psychiatry, 43, 665-668.

Krystkowiak, P., Martinat, P., Cassim, F., Pruvo, J.-P., Leys, D., Guieu, J.-D., et al. (2000). Thalamic tremor: correlations with three-dimensional magnetic resonance imaging data and pathophysiological mechanisms. Movement Disorders, 15(5), 911-918.

Krystkowiak, P., Martinat, P., Defebvre, L., Pruvo, J. P., Leys, D., \& Destee, A. (1998).

Dystonia after striatopallidal and thalamic stroke: clinicoradiological correlations and pathophysiological mechanisms. Journal of Neurology, Neurosurgery and Psychiatry, 65(5), 703-708. 
Lang, D. J., Kopala, L. C., Vandorpe, R. A., Rui, Q., Smith, G. N., Goghari, V. M., et al. (2001). An MRI study of basal ganglia volumes in first-episode schizophrenia patients treated with risperidone. American Journal of Psychiatry, 158(4), 625-631.

LeDoux, M. S., \& Brady, K. A. (2003). Secondary cervical dystonia associated with structural lesions of the central nervous system. Movement Disorders, 18(1), 60-69.

Lehericy, S., Vidailhet, M., Dormont, D., Pierot, L., Chiras, J., Mazetti, P., et al. (1996).

Striatopallidal and thalamic dystonia: a magnetic resonance imaging anatomoclinical study. Archives of Neurology, 53(3), 241-250.

Lenz, F. A., Jaeger, J. C., Seike, M. S., Lin, Y. C., \& Reich, S. G. (2002). Single-neuron analysis of human thalamus in patients with intention tremor and other clinical signs of cerebellar disease. Journal of Neurophysiology, 87, 2084-2094.

Lozza, C., Marie, R.-M., \& Baron, J.-C. (2002). The metabolic substrates of bradykinesia and tremor in uncomplicated Parkinson's disease. NeuroImage, 17, 688-699.

MacDonald, A. W., Pogue-Geile, M. F., Debski, T. T., \& Manuck, S. (2001). Genetic and environmental influences on schizotypy: a community-based twin study. Schizophrenia Bulletin, 27(1), 47-58.

Marcelis, M., Suckling, J., Woodruff, P., Hofman, P., Bullmore, E., \& van Os, J. (2003). Searching for a structural endophenotype in psychosis using computational morphometry. Psychiatry Research, 122(3), 153-167.

Marsden, C. D., \& Jenner, P. (1980). The pathophysiology of extrapyramidal side-effects of neuroleptic drugs. Psychological Medicine, 10, 55-72.

McCreadie, R. G., \& Ohaeri, J. U. (1994). Movement disorder in never and minimally treated Nigerian schizophrenic patients. British Journal of Psychiatry, 164, 184-189. 
McCreadie, R. G., Thara, R., Kamath, S., Padmavathi, R., Latha, S., Menon, M. S., et al. (1996). Abnormal movements in never-medicated Indian patients with schizophrenia. The British Journal of Psychiatry, 168(2), 221-226.

McCreadie, R. G., Thara, R., Padmavati, R., Srinivasan, T. N., \& Jaipurkar, S. D. (2002).

Structural brain differences between never-treated patients with schizophrenia, with and without dyskinesia, and normal control subjects: a magnetic resonance imaging study. Archives of General Psychiatry, 59(4), 332-336.

McCreadie, R. G., Thara, R., Srinivasan, T. N., \& Padmavathi, R. (2003). Spontaneous dyskinesia in first-degree relatives of chronically ill, never-treated people with schizophrenia. British Journal of Psychiatry, 183, 45-49.

Menon, V., Anagnoson, R. T., Glover, G. H., \& Pfefferbaum, A. (2001). Functional magnetic resonance imaging evidence for disrupted basal ganglia function in schizophrenia. American Journal of Psychiatry, 158(4), 646-649.

Muller, J. L., Roder, C., Schuierer, G., \& Klein, H. E. (2002). Subcortical overactivation in untreated schizophrenic patients: a functional magnetic resonance image finger-tapping study. Psychiatry and Clinical Neurosciences, 56(1), 77-84.

Naumann, M., Becker, G., Toyka, K. V., Supprian, T., \& Reiners, K. (1996). Lenticular nucleus lesion in idiopathic dystonia detected by transcranial sonography. Neurology, 47(5), 1284-1290.

Noldus. (2003). The Observer Video Pro (Version 5.0). Leesburg, VA: Noldus Information Technology, Inc.

O'Donovan, M. C., Williams, N. M., \& Owen, M. J. (2003). Recent advances in the genetics of schizophrenia. Human Molecular Genetics, 12(2), R125-R133. 
Owens, D. G. C., Johnstone, E. C., \& Frith, C. D. (1982). Spontaneous involuntary disorders of movement. Archives of General Psychiatry, 39, 452-461.

Perlmutter, J. S., Stambuk, M. K., Markham, J., Black, K. J., McGee-Minnich, L., Jankovic, J., et al. (1997). Decreased [18F]spiperone binding in putamen in idiopathic focal dystonia. The Journal of Neuroscience, 17(2), 843-850.

Pfohl, B., Stangl, D., \& Zimmerman, M. (1982). The Structured Interview for DSM-III Personality Disorders (SIDP).Iowa City, IA: University of Iowa Department of Psychiatry.

Pogue-Geile, M. F. (in press). Schizophrenia Spectrum Disorders. In Encyclopedia of the Human Genome: Nature Publishing Group.

Pogue-Geile, M. F., \& Gottesman, I. I. (1999). Schizophrenia: Study of a Genetically Complex Phenotype. In B. C. Jones \& P. Mormede (Eds.), Neurobehavioral Genetics: Methods and Applications. New York, NY: CRC Press.

Puri, B. K., Barnes, T. R. E., Chapman, M. J., Hutton, S. B., \& Joyce, E. M. (1999). Spontaneous dyskinesia in first episode schizophrenia. Journal of Neurology, Neurosurgery and Psychiatry, 66(1), 76-78.

Sigmundsson, T., Suckling, J., Maier, M., Williams, S., Bullmore, E., Greenwood, K., et al. (2001). Structural abnormalities in frontal, temporal, and limbic regions and interconnecting white matter tracts in schizophrenic patients with prominent negative symptoms. American Journal of Psychiatry, 158(2), 234-243.

Spitzer, R. L., Endicott, J., \& Robins, E. (1978). Research diagnostic criteria: Rationale and reliability. Archives of General Psychiatry, 35, 773-782. 
Srinivasan, T. N., Thara, R., Padmavathi, R., \& McCreadie, R. G. (2001). Relationship of extrapyramidal symptoms to age at onset and drug treatment in middle-aged and elderly schizophrenic patients. Schizophrenia Research, 47, 69-75.

Staal, W. G., Hulshoff Pol, H. E., Schnack, H. G., Hoogendoorn, M. L., Jellema, K., \& Kahn, R. S. (2000). Structural brain abnormalities in patients with schizophrenia and their healthy siblings. American Journal of Psychiatry, 157(3), 416-421.

Staal, W. G., Hulshoff Pol, H. E., Schnack, H. G., van Haren, N. E., Seifert, N., \& Kahn, R. S. (2001). Structural brain abnormalities in chronic schizophrenia at the extremes of the outcome spectrum. American Journal of Psychiatry, 158(7), 1140-1142.

Walker, E., Lewis, N., Loewy, R., \& Palyo, S. (1999). Motor dysfunction and risk for schizophrenia. Development and Psychopathology, 11, 509-523.

Woods, B., Kinney, D., \& Yurgelun-Todd, D. (1986). Neurologic abnormalities in schizophrenic patients and their families I. Comparison of schizophrenic, bipolar, and substance abuse patients and normal controls. Archives of General Psychiatry, 43, 657-663.

Yazici, A. H., Demir, B., Yazici, K. M., \& Gogus, A. (2002). Neurological soft signs in schizophrenic patients and their nonpsychotic siblings. Schizophrenia Research, 58, 241246.

Young, K. A., Manaye, K. F., Liang, C., Hicks, P. B., \& German, D. C. (2000). Reduced number of mediodorsal and anterior thalamic neurons in schizophrenia. Biological Psychiatry, 47(11), 944-953. 